\title{
Estudio Numérico de Flujos Turbulentos Isotérmicos en Canales y Flujos Laminares con Convección Mixta en Cavidades
}

\author{
Elizaldo D. dos Santos, Carla M. Xavier, Adriane P. Petry \\ Universidad Federal del Rio Grande del Sul, Programa de Posgrado en Ingeniería Mecánica, \\ Calle Sarmento Leite, 425, CEP: 90.050-170 Porto Alegre, RS-Brasil. \\ (e-mail: edsantos@mecanica.ufrgs.br; xavier_carla@yahoo.com.br; adrianep@mecanica.ufrgs.br) \\ Recibido Mar. 31, 2010; Aceptado May. 24, 2010; Versión Final recibida Jul. 23, 2010
}

\section{Resumen}

Se ha realizado un estudio numérico sobre flujos estacionarios turbulentos, en canales tridimensionales y flujos transitorios laminares en cavidades con transferencia de calor por convección mixta. Las ecuaciones de conservación se resuelven a través del Método de Elementos Finitos utilizando esquema temporal explicito de Taylor-Galerkin. La simulación de Grandes Escalas se emplea para el tratamiento de la turbulencia. Para el caso isotérmico, flujos con $\operatorname{Re}=3300$ son simulados usando los modelos submalla de Smagorinsky y Dinámico. Este último modelo permitió mejorar los perfiles de velocidad media y las estadísticas de la turbulencia. Los campos transitorios de velocidad y temperatura se compararon con los resultados de la literatura, obteniéndose un desvío inferior a $6 \%$.

Palabras clave: flujo turbulento, flujo laminar, convección mixta, cavidades, métodos numéricos

\section{Numerical Study of Isothermal Turbulent Channel Flows and Mixed Convection Laminar Cavity Flows}

\begin{abstract}
A numerical study about three-dimensional steady state turbulent channel flows and laminar transient cavity flows with mixed convection heat transfer has been done. The solution of the conservation equations is obtained by means of Finite Element Method and Taylor-Galerkin explicit scheme. Large Eddy Simulation is employed for the treatment of turbulence. For the isothermal case, flows with $\operatorname{Re}=3300$ were simulated using the Smagorinsky and Dynamical subgrid models. The latter model allowed improving the average velocity profiles as well as turbulence statistics. The transient velocity and temperature fields were compared with results of the literature, leading to a deviation lower than $6 \%$.
\end{abstract}

Keywords: turbulent flow, laminar flow, mixed convection, cavities, numerical methods 


\section{INTRODUCCIÓN}

Flujos turbulentos con transferencia de calor se presentan en varios fenómenos en el campo de la ingeniería (intercambiadores de calor, generadores de vapor, enfriamiento de paquetes electrónicos entre otros) y en los fenómenos naturales (dispersión de contaminantes en la atmósfera). La conexión entre los fenómenos de turbulencia y transferencia de calor ha sido una fuerte motivación para el entendimiento del proceso dinámico en la región de la superficie y ha llevado a muchas investigaciones numéricas y experimentales (Kobayashi, 2006; Eiamsa-ard and Promvonge, 2009). En el presente trabajo se enfoca el mecanismo de transferencia de calor por convección mixta.

Una herramienta poderosa es el análisis numérico de las ecuaciones gobernantes (conservación de masa, cantidad de movimiento y energía) que modelan matemáticamente flujos incompresibles, turbulentos y no isotérmicos. De entre los diferentes métodos numéricos existentes, se optó por utilizar el método de elementos finitos (FEM) que, según Reddy y Gartling (1994), posee la ventaja de hábilmente representar dominios complejos para la solución de problemas de contorno e iniciales crecientes en el campo de la ingeniería, tornándolo una herramienta práctica.

La gran variación existente entre las grandes y las pequeñas escalas presentes en la estructura de la turbulencia, asociada con sus características como: irregularidad, elevados números de Reynolds y la tridimensionalidad de las estructuras vorticiales tornan el análisis numérico de este fenómeno, a través del abordaje numérico de Simulación Directa, extremamente onerosa, visto que los dominios bajo análisis precisan de enormes discretizaciones espaciales y temporales, conduciendo a un número de grados de libertad y de ecuaciones muy grande para ser resueltas en cualquier equipamiento computacional disponible actualmente. Según Wang et al. (2005) el número de nodos de una malla para simulación de un flujo no-isotérmico es del orden de $\operatorname{Pr}^{3} \operatorname{Re}^{9 / 4}$. Algunos ejemplos del costo computacional correlacionado con la simulación directa pueden ser vistos en (Lesieur et al., 2005).

Como consecuencia de la imposibilidad de aplicar Simulación Directa para la gran mayoría de los problemas es necesario utilizar metodologías alternativas como los modelos clásicos basados en la hipótesis de la media temporal de Reynolds (RANS) (Wilcox, 2002) y en la Simulación de Grandes Escalas (LES) utilizada en el presente trabajo (Lesieur et al., 2005; Saugat, 2006). En la Simulación de Grandes Escalas se realiza solamente el modelado de las pequeñas escalas (siendo las grandes escalas resueltas directamente). La gran ventaja de utilizar este tipo de abordaje es que las escalas submalla poseen una naturaleza más universal, con una estructura más isotrópica, del que las restantes estructuras turbulentas (Lesieur et al., 2005; Saugat, 2006) lo que permite un modelado más adecuado del flujo, además de requerir un menor costo computacional que la simulación directa.

El modelado numérico de flujos incompresibles a través del método de elementos finitos presenta dificultades oriundas de las fuertes características del problema discretizado, causadas por la presencia de elementos nulos en la diagonal principal de la matriz de masa correspondiente a la ausencia de los términos de presión (de forma explícita) en la ecuación de la continuidad. Puede ser citado como ejemplo la imposibilidad del uso de métodos de integración temporales puramente explícitos debido a la ausencia de la derivada temporal de la presión en la ecuación de la continuidad (Reddy y Gartling, 1994). Con el objetivo de superar esa dificultad es usado el método de seudo-compresibilidad (Kawahara y Hirano, 1983) donde la derivada de la presión en el tiempo permanece en la ecuación de la continuidad (Petry y Awruch, 2006).

En el presente trabajo fueron implementados los términos de la ecuación de la energía y de flotabilidad en la ecuación de la conservación de la cantidad de movimiento en un código desarrollado en FORTRAN para simulación de flujos tridimensionales, casi-incompresibles e isotérmicos desarrollados por (Petry, 2002). El método numérico utilizado fue el de elementos finitos con elementos hexaédricos trilineares de ocho nodos, la discretización espacial fue hecha utilizando el método estándar de Galerkin (Reddy y Gartling, 1994). En lo que respecta al análisis 
de los términos transitorios fue utilizado el esquema temporal explícito de Taylor-Galerkin (Donea, 1984; Yoon et al., 1998; Zienkiewicz y Taylor, 2000).

Fueron obtenidos resultados de los campos de velocidad y temperatura, analizándose la variación temporal y espacial, para un flujo con número de $\operatorname{Re}=400$ y $\mathrm{Gr}=16000$, tales resultados fueron comparados a los resultados numéricos presentados por (Ji et al., 2007). También fue realizado un comparativo entre los modelos submalla clásicos de Smagorinsky (Smagorinsky, 1963) y dinámico (Germano et al., 1991; Lilly, 1992) para determinación del perfil de velocidad de flujos turbulentos, tridimensionales e isotérmicos en canales, en el modelo dinámico es utilizada la metodología desarrollada por (Petry, 2002) llamada segundo filtrado por elementos finitos independientes.

\section{ASPECTOS MATEMÁTICOS Y NUMÉRICOS}

Para la simulación de flujos turbulentos, tridimensionales, transitorios y no isotérmicos utilizando LES es necesaria la realización de un proceso de filtrado para la separación de las escalas (Lesieur et al., 2005; Saugat, 2006) en las ecuaciones de conservación de masa, cantidad de movimiento y energía conforme muestra la Ec. 1:

$v_{i}=\bar{v}_{i}+v_{i}^{\prime} \quad p=\bar{p}+p^{\prime} \quad \rho=\bar{\rho}+\rho^{\prime} \quad T=\bar{T}+T^{\prime}$

Donde la barra sobre la variable indica el campo de grandes escalas, el apostrofe indica un campo submalla. Todas las propiedades termo físicas serán evaluadas en el campo medio (grandes escalas), entonces $\rho^{\prime}=C_{P}{ }^{\prime}=k^{\prime}=0$. Después el proceso de filtrado las ecuaciones gobernantes (conservación de masa, cantidad de movimiento y energía, respectivamente) quedan de la siguiente forma, Ec. 2, 3 y 4 :

$$
\begin{aligned}
& \frac{\partial \bar{P}}{\partial t}+C^{2} \frac{\partial}{\partial x_{j}}\left(\rho_{0} \bar{v}_{j}\right)=0 \\
& \frac{\partial}{\partial t}\left(\rho_{0} \bar{v}_{i}\right)+\frac{\partial}{\partial x_{j}}\left(\rho_{0} \bar{v}_{i} \bar{v}_{j}\right)+\frac{\partial P}{\partial x_{j}} \delta_{i j}-\frac{\partial}{\partial x_{j}}\left\{U\left(\frac{\partial}{\partial x_{j}}\left(\rho_{0} \bar{v}_{i}\right)+\frac{\partial}{\partial x_{i}}\left(\rho_{0} \bar{v}_{j}\right)\right)+\frac{\lambda}{\rho_{0}}\left(\frac{\partial}{\partial x_{k}}\left(\rho_{0} \bar{v}_{k}\right)\right) \delta_{i j}\right\}+{ }_{(\mathrm{i}, \mathrm{j}, \mathrm{k}=1,2 \text { y } 3) \text { en } \mathrm{t} x \Omega} \Omega \\
& +\frac{\partial}{\partial x_{j}}\left\{\rho_{0}\left(L_{i j}+C_{i j}+\bar{v}_{i}^{\prime} v_{j}^{\prime}\right)\right\}+\rho_{0} g_{i} \beta\left(\bar{T}-T_{0}\right)=0
\end{aligned}
$$

$$
\frac{\partial \bar{T}}{\partial t}+\frac{\partial}{\partial x_{j}}\left(\bar{v}_{j} \bar{T}\right)=\frac{\partial}{\partial x_{j}}\left[\alpha(T) \frac{\partial \bar{T}}{\partial x_{j}}-\left(\theta_{j}+C_{\theta j}+L_{\theta j}\right)\right]+q^{\prime \prime \prime} \quad(\mathrm{i}, \mathrm{j}, \mathrm{k}=1,2 \text { y } 3) \text { en } \mathrm{t} \times \Omega
$$

Con las siguientes condiciones de contorno e iniciales:

$$
\begin{array}{llr}
v_{i}=\hat{v}_{i} \quad T=\hat{T} & (\mathrm{i}=1,2 \text { y } 3) \text { en } \Gamma_{\mathrm{D}} \\
\left(\sigma_{i j}-p \delta_{i j}\right) n_{j}=t_{i} & \left(k \partial_{x_{j}} T\right) n_{j}+q_{c}+q_{r}=q & (\mathrm{i}, \mathrm{j}=1,2 \text { y } 3) \text { en } \Gamma_{\mathrm{N}} \\
v_{i}=\hat{v}_{i 0} \quad p=\hat{p}_{i 0} & T=\hat{T}_{i 0} & \text { en } \mathrm{t}=0, \Omega
\end{array}
$$


Las propiedades termo físicas, aunque escritas como función de la temperatura, serán consideradas constantes en el presente trabajo para facilitar el modelado del problema (evitando no linealidades insertadas en las ecuaciones). En lo que se refiere a los términos submalla, los mismos pueden ser identificados: $T_{i j}=\overline{v_{i} v_{j}^{\prime}}$ (tensiones de Reynolds submalla); $C_{i j}=\overline{v_{i}^{\prime} \overline{v_{j}}}+\overline{\overline{v_{i}} v_{j}^{\prime}}$ (términos cruzados); $L_{i j}=\overline{\overline{v_{i}}} \overline{v_{j}}-\overline{v_{i}} \overline{v_{j}}$ (términos de Leonard); $\theta_{j}=\overline{v_{i}^{\prime} T^{\prime}}$ (flujo turbulento submalla); $C_{\theta j}=\overline{\overline{v_{j}} T^{\prime}}+\overline{v_{j}^{\prime} T^{\prime}}$ (flujo turbulento cruzado); $L_{\theta j}=\overline{\overline{v_{j}}} \overline{\bar{T}}+\overline{v_{j}} \bar{T}$ (flujo turbulento de Leonard).

En general los términos $L_{i j}$ y $C_{i j}$ pueden ser despreciados (Lesieur et al., 2005). Inclusive, en el trabajo de (Silveira Neto et al., 1993) fue realizada una comparación entre las tensiones de Reynolds submalla y la suma de los términos cruzados y de Leonard $\left(L_{i j}+C_{i j}\right)$, donde aquellas fueron más de cuarenta veces mayores que estas. En lo que se refiere a los términos de flujo turbulento de Leonard y cruzado, Silveira Neto et al. (1993) despreciaron estos términos en su trabajo. Teniendo en cuenta lo que fue dicho antes, también despreciaremos los términos y flujos turbulentos cruzados y de Leonard.

\section{Modelos submalla}

Los dos modelos implementados son basados en el concepto de viscosidad turbulenta. Usando la hipótesis de Boussinesq, las tensiones de Reynolds submalla son dadas por la Ec. 8:

$$
-\overline{v_{i}^{\prime} v_{j}^{\prime}}=u_{T}\left(\partial \bar{v}_{i} / \partial x_{j}+\partial \bar{v}_{j} / \partial x_{i}\right)
$$

El modelo para el flujo turbulento es obtenido por analogía entre el transporte de cantidad de movimiento y energía. Consecuentemente, la misma hipótesis de Boussinesq es aplicada al flujo turbulento submalla el cual está dado por Ec.9:

$$
-\overline{v_{j}^{\prime} T^{\prime}}=\alpha_{T} \partial \bar{T} / \partial x_{j}
$$

Recordando que la Ec. 10 no requiere de ninguna modificación (inserción de términos relacionados a la energía cinética submalla) pues la ecuación de la continuidad fue modificada para flujos casi incompresibles.

\section{Modelo de Smagorinsky}

El modelo de Smagorinsky (Smagorinsky, 1963) es el primer modelo submalla introducido para la determinación de las tensiones de Reynolds submalla y del flujo turbulento submalla y aún es bastante utilizado. En este modelo la viscosidad turbulenta es dada por:

$$
U_{T}=C_{s}^{2} \bar{\Delta}^{2}|\bar{S}|
$$

Donde $\mathrm{C}_{\mathrm{S}}$ es la constante de Smagorinsky (que varía de 0,1 a 0,23 aproximadamente), $\bar{\Delta}$ es la longitud característica, dada por la Ec. 11, $|\bar{S}|$ es dado por la Ec. 12 y el tensor de deformación $\overline{S_{i j}}$ es dado por la Ec. 13.

$$
\bar{\Delta}=\sqrt[3]{\prod_{i=1}^{3} \Delta x_{i}}
$$




$$
\begin{aligned}
& |\bar{S}|=\sqrt{2 \bar{S}_{i j} \bar{S}_{i j}} \\
& \bar{S}_{i j}=\frac{1}{2}\left(\partial \bar{v}_{i} / \partial x_{j}+\partial \bar{v}_{i} / \partial x_{i}\right)
\end{aligned}
$$

Y la difusividad turbulenta es indirectamente determinada por la razón de la viscosidad turbulenta y del número de Prandtl turbulento, dado por la siguiente expresión:

$\alpha_{T}=C_{s}^{2} / \operatorname{Pr}_{T} \bar{\Delta}^{2}|\bar{S}|$

El modelo dinámico submalla propuesto por (Germano et al., 1991) y modificado por (Lilly, 1992) fue implementado para la solución del campo hidrodinámico (todavía no lo fue para el campo térmico). El tensor de Reynolds submalla también es aproximado por la Ec. 8, considerando todo esto la viscosidad turbulenta está definida como:

$$
v_{t}=C(\mathbf{x}, t) \bar{\Delta}^{2}\left|\bar{S}_{i j}\right|
$$

El coeficiente dinámico es calculado en función a las características locales del flujo, utilizando un proceso de doble filtrado. El cálculo de $\mathrm{C}(\mathrm{x}, \mathrm{t})$ está basado en las informaciones de las menores escalas resueltas, siendo definido por la Ec. 16 y los tensores $L_{i j}$ y $M_{i j}$ relacionados con esta ecuación son presentados en la Ec. 17:

$$
\begin{aligned}
& C(\mathbf{x}, t)=-\frac{1}{2} \frac{L_{i j} M_{i j}}{M_{i j} M_{i j}} \\
& L_{i j}=\left\langle\bar{v}_{i} \bar{v}_{j}\right\rangle-\left\langle\bar{v}_{i}\right\rangle\left\langle\bar{v}_{j}\right\rangle \quad M_{i j}=\langle\bar{\Delta}\rangle^{2}\left|\left\langle\bar{S}_{i j}\right\rangle\right|\left\langle\bar{S}_{i j}\right\rangle-\left\langle\bar{\Delta}^{2}\left|\bar{S}_{i j}\right| \bar{S}_{i j}\right\rangle \\
& \left\langle\bar{S}_{i j}\right\rangle=\frac{1}{2}\left(\partial\left\langle\bar{v}_{i}\right\rangle / \partial x_{j}+\partial\left\langle\bar{v}_{j}\right\rangle / \partial x_{i}\right) \quad|\langle\bar{S}\rangle|=\sqrt{2\left\langle\bar{S}_{i j}\right\rangle\left\langle\bar{S}_{i j}\right\rangle}
\end{aligned}
$$

Donde $\langle\bar{\Delta}\rangle$ define la longitud característica del segundo filtro, siendo $\langle\bar{\Delta}\rangle>\bar{\Delta}$. En las ecuaciones anteriores, la barra indica el primer proceso de filtrado (filtro a nivel de malla) y el símbolo \langle\rangle se refiere al segundo proceso de filtrado (filtro de prueba).

Análisis numérico

Para la solución del sistema de ecuaciones, el método de elementos finitos es empleado. Para obtener el sistema de ecuaciones algébricas, las derivadas temporales son expandidas en series de Taylor, incluyendo los términos de segunda orden (Zienkiewicz y Taylor, 2000) y para la discretización espacial el método estándar de Galerkin es aplicado (Reddy y Gartling, 1994). Para ahorrar tiempo de procesamiento son utilizadas expresiones analíticas para las matrices y elementos hexaédricos isoparamétricos (Azevedo, 1999). El esquema temporal es explícito y condicionalmente estable; y el paso de integración posee la siguiente restricción:

$$
\Delta t \leq \Delta x_{i}(\min ) / C+V
$$

Donde $\Delta x_{i}$ (min) es la dimensión mínima de los elementos de la malla, $C$ es la velocidad del sonido en el medio y $\vee$ es la velocidad de referencia. 
Para la realización del segundo filtrado, utilizada en la determinación del coeficiente $C(x, t)$ del modelo dinámico, fue utilizada la metodología propuesta por (Petry, 2002). El Segundo Filtrado por Elementos Finitos utiliza técnicas comunes al método de elementos finitos, como: la definición de los elementos por conectividades, el uso de dos sistemas de coordenadas (global $\left(\mathrm{x}_{1}, \mathrm{x}_{2}, \mathrm{x}_{3}\right)$ y natural $(\xi, \eta, \zeta))$ la transformación de coordenadas y funciones de interpolación de elementos. El esquema consiste en la generación de un súper elemento en torno de cada nodo de la malla, entonces, con funciones de forma usuales, una interpolación linear de las variables en los nodos de los súper elementos es realizada para adquirir valores filtrados en el correspondiente nodo interno. Mayores detalles sobre esa metodología pueden ser vistos en (Petry, 2002).

\section{RESULTADOS}

Canal isotérmico $(\mathrm{Re}=3300)$

Simulaciones de flujo en canal tridimensional con número de Reynolds 3300 son realizadas para validar el código. Los resultados son obtenidos usando los modelos de Smagorinsky y Dinámico y comparados con datos experimentales (Nishino y Kasagi, 1989) y con otras simulaciones numéricas (Kim et al., 1987; Germano et al., 1991). La geometría del canal y la malla uniforme de $60 \times 60 \times 20$ elementos en las direcciones $(x, y, z)$ son mostradas, respectivamente, en las Fig. 1 y 2.

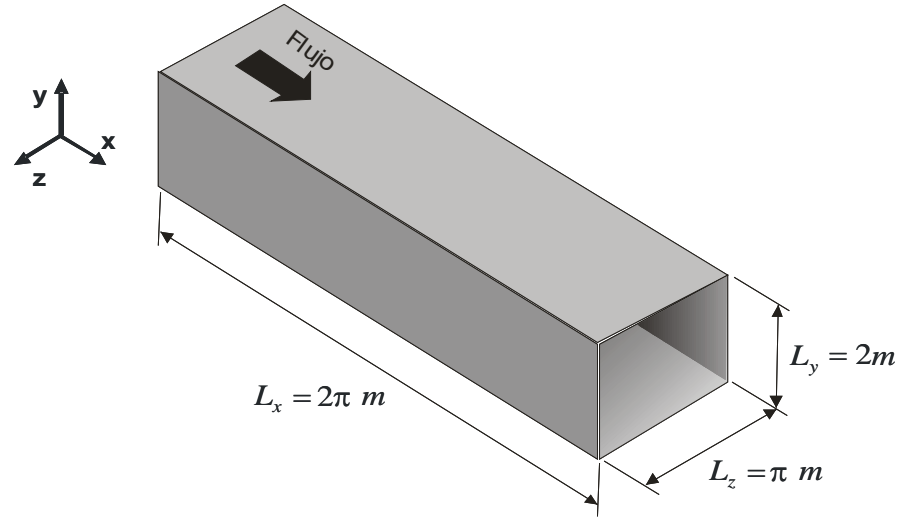

Fig. 1: geometría del canal

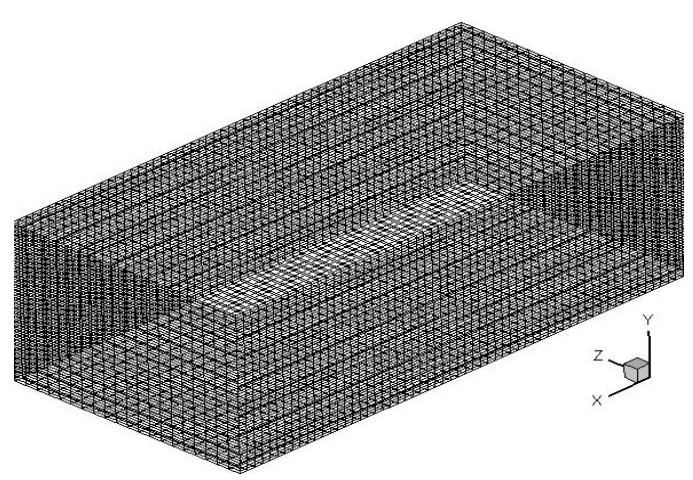

Fig. 2: malla uniforme

Como condiciones de contorno, en la entrada se usa un perfil turbulento de velocidad, completamente desarrollados $\left(\mathrm{v}_{1}=\mathrm{V}(\mathrm{y}), \mathrm{v}_{2}=0\right)$ y condición de no-deslizamiento $\left(\mathrm{v}_{1}=\mathrm{v}_{2}=\mathrm{v}_{3}=0\right)$ preescrita en las paredes superior y inferior. En la salida del canal existen condiciones de contorno naturales $\left(t_{1}=t_{2}=t_{3}=0\right.$ ) (vea la Ec.5). Las condiciones iniciales utilizadas son $v_{1}=50,49 \mathrm{~m} / \mathrm{s}$, $v_{2}=v_{3}=p=0$. Es importante observar que, diferentemente del procedimiento normal adoptado en simulaciones numéricas de flujos turbulentos en canales con DNS o LES, las condiciones de contorno en las direcciones $x$ y $z$ en el serán periódicas. Además, en la simulación usando el modelo submalla de Smagorinsky es usado el valor $C_{S}=0,44$, que es sugerido por Abrunhosa (2003) para la simulación de canales.

En la Fig. 3 son mostrados los resultados de la simulación, en los modelos de Smagorinsky y Dinámico, del perfil de velocidad $V_{1}$ comparados con resultados experimentales y otras simulaciones. La tensión de Reynolds ( $\left.u^{\prime} v^{\prime}\right)$ estimada por los modelos es mostrada en la Fig. 4, estas tensiones fueran comparadas con los resultados de (Kim et al., 1987). 


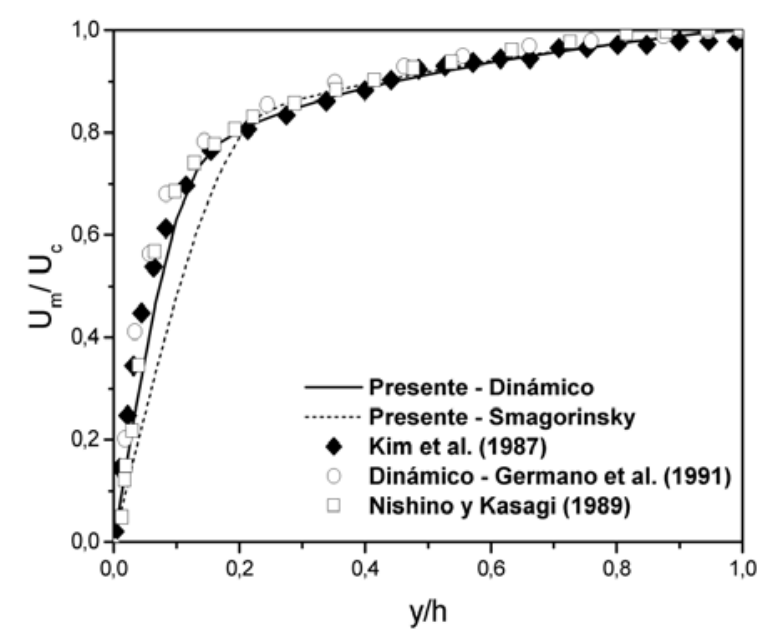

Fig. 3: Perfil de velocidad U

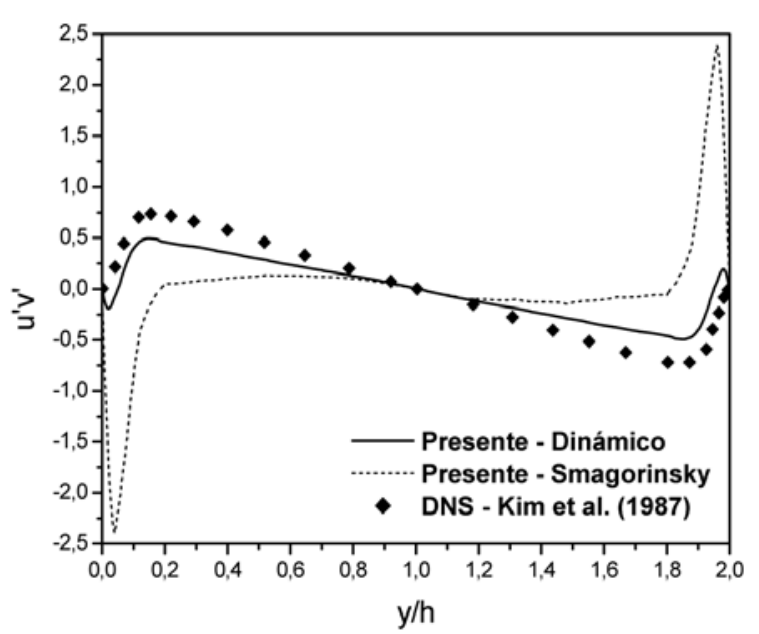

Fig. 4: Tensión de Reynolds u'v'

El coeficiente de fricción, basado en la velocidad volumétrica media del canal, obtenido por (Kim et al., 1987) fue de $C_{f 0}=6,04 \times 10^{-4}$. En el presente trabajo, tanto el coeficiente obtenido con el modelo dinámico cuanto el obtenido con el modelo de Smagorinsky fueran más bajos, con una diferencia de $4,5 \%$ y $16 \%$, respectivamente. Con referencia a las estadísticas de la turbulencia, las tensiones no se previeron de manera satisfactoria con el modelo de Smagorinsky, lo que sugiere que la constante del modelo debería haber sido menor. Las tensiones obtenidas a través del modelo dinámico con la misma malla empleada en el modelo de Smagorinsky llevaron a resultados más satisfactorios, reduciendo los problemas relacionados a selección del parámetro submalla. Así las tensiones para este modelo también fueron ligeramente sobrestimadas. Entonces, es posible decir que la selección adecuada del parámetro submalla, que es realizada por el modelo dinámico, llevó a la mejora de los parámetros medios (velocidad media y coeficiente de fricción) así como de las estadísticas de la turbulencia.

Cavidad no isotérmica $(\operatorname{Re}=400)$

En este tópico, se presentan los resultados de flujos bidimensionales, incompresibles y no isotérmicos en régimen laminar para número de Reynolds $R_{H_{H}}=400$ y de Grashoff $\mathrm{Gr}=16000$ en cavidad forzada con una de las superficies calentadas, conforme fue visto en el dominio en la Fig. 5. Los resultados aquí obtenidos son comparados con los resultados numéricos presentados en (Ji et al., 2007) según este autor, el código que generó los resultados numéricos presentados en este trabajo fue validado con resultados experimentales realizados por el mismo. La malla utilizada para la discretización del dominio fue de $100 \times 100 \times 1$ con mayor refinamiento junto a las paredes. Simulación de Grandes Escalas también es empleada en esta simulación, lo mismo para el estudio de un flujo laminar. En este caso los parámetros submalla utilizados fueron $C_{S}=0,1$ y $\operatorname{Pr}_{\mathrm{T}}=0,9$, que son sugeridos por (Ampofos y Karayiannis, 2003).

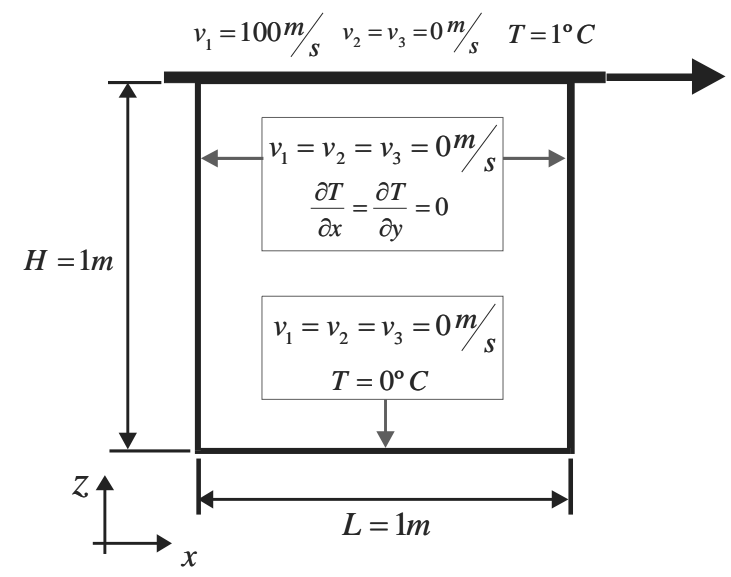

Fig.5: Dominio de la cavidad forzada con sus condiciones de contorno adimensionales 
Las condiciones de contorno e iniciales utilizadas en el presente trabajo fueron las mismas utilizadas en (Ji et al., 2007), las de contorno pueden ser vistas en la Fig. 5 en cuanto las iniciales son reescritas abajo:

$V_{1}(x, y, z, t=0)=0 \quad V_{2}(x, y, z, t=0)=0 \quad T^{*}(x, y, z, t=0)=Z^{*}$

En la Fig. 6 son presentadas isotermas, permitiendo una comparación entre los campos de temperatura obtenidos por (Ji et al., 2007) (Fig. 6 - a, b y c) y los obtenidos en el presente trabajo (Fig. $6 \mathrm{~d}$, e y f) para los tiempos adimensionales de $t_{a d m}=1.4,3.7$ y 5 respectivamente. Las isotermas demuestran que los dos métodos numéricos utilizados presentan un comportamiento bastante semejante en el régimen transitorio.

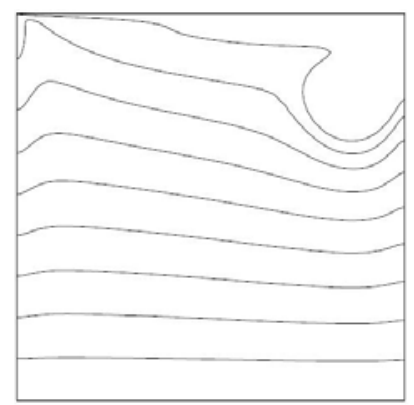

a)

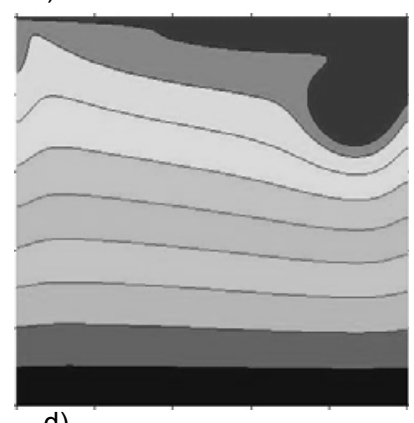

d) $\quad t_{\text {adm }}=1.4$

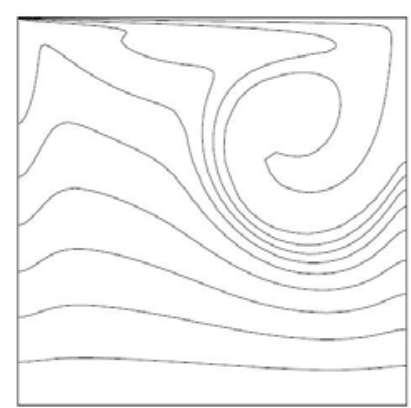

b)

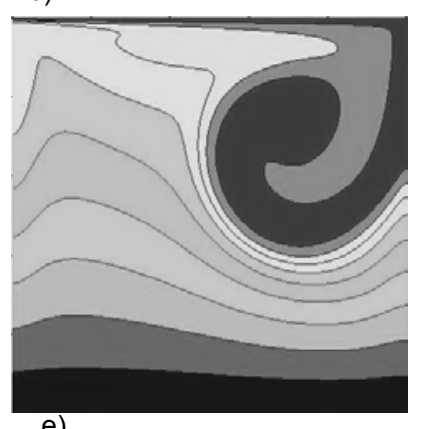

e) $\quad t_{\mathrm{adm}}=3.7$

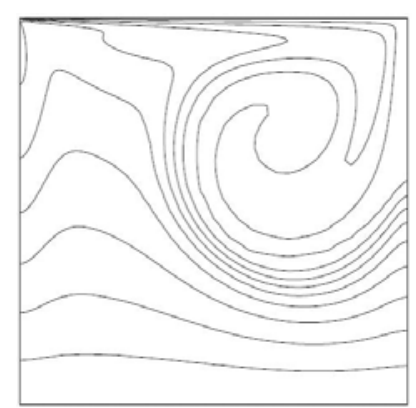

c)

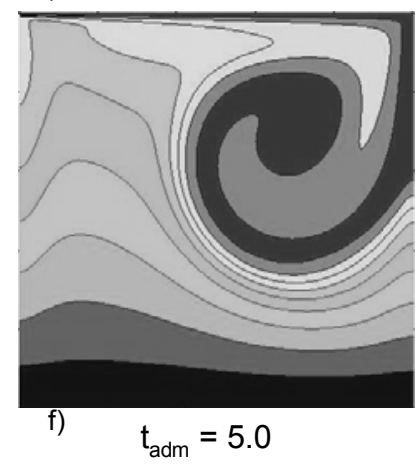

Fig. 6: Campos de temperatura transitorios - resultados numéricos de (Ji et al., 2007) a) $t_{a d m}=1.4$, b) $t_{a d m}=3.7$, c) $t_{a d m}=1.4$ - resultados numéricos del presente trabajo d) $\left.t_{a d m}=1.4, e\right) t_{a d m}=3.7$ e f)

$$
\mathrm{t}_{\mathrm{adm}}=5.0
$$

En la Fig. 7 es posible evaluar la evolución de la temperatura (Fig. 7a) y velocidad en X (Fig. 7b) con el tiempo (adimensionales) en el plano vertical medio $(X=0,5)$ para un valor fijo de $Z=0,27$ para el presente código sin la utilización de los términos de flotabilidad en la formulación (denominado en el gráfico: convección _ forzada) y con los términos de flotabilidad insertados (convección_mixta), ya en las Fig. 8 y 9 (a - campo de temperaturas y b-campo de velocidades en $x$ ) son presentados los resultados para dos posiciones de la cavidad en la coordenada $Z$ $(Z=0,48$, próximo al centro de la cavidad y $Z=0,93$ próximo a placa móvil). En $Z=0,27$ fue donde los resultados obtenidos por el código desarrollado en el presente trabajo presentan mayor discordancia con los resultados de ( $\mathrm{Ji}$ et al., 2007) (que utiliza el método de volúmenes finitos basado en el algoritmo SIMPLER), obteniéndose un error medio estadístico porcentual de $\varepsilon=$ $5,56 \%$ en esta región de la cavidad para el programa con flotabilidad (consecuentemente, menor que el error de medición I $\sim 7,1 \%$ presentado por el autor para el experimento). De un modo general los resultados obtenidos en el presente trabajo se presentaran muy semejantes a los obtenidos por (Ji et al., 2007) permitiendo una validación del presente código desarrollado para casos no isotérmicos en régimen laminar. En todas las regiones donde los campos de velocidad y temperatura fueron comparados se nota la existencia de una tendencia de convergencia de los resultados conforme el flujo tiende al régimen permanente. También se observa la mejora en el campo de temperaturas y en el perfil de velocidades al insertar los términos de flotabilidad en las ecuaciones de conservación de cantidad de movimiento. 
Para obtener los resultados fue utilizado el supercomputador SunFire X2200, AMD Opteron 1.8GHz Dual Core del Centro Nacional de Supercomputación (CESUP-RS). Para el mejoramiento del desempeño computacional fue utilizada la técnica de paralelización OpenMP.
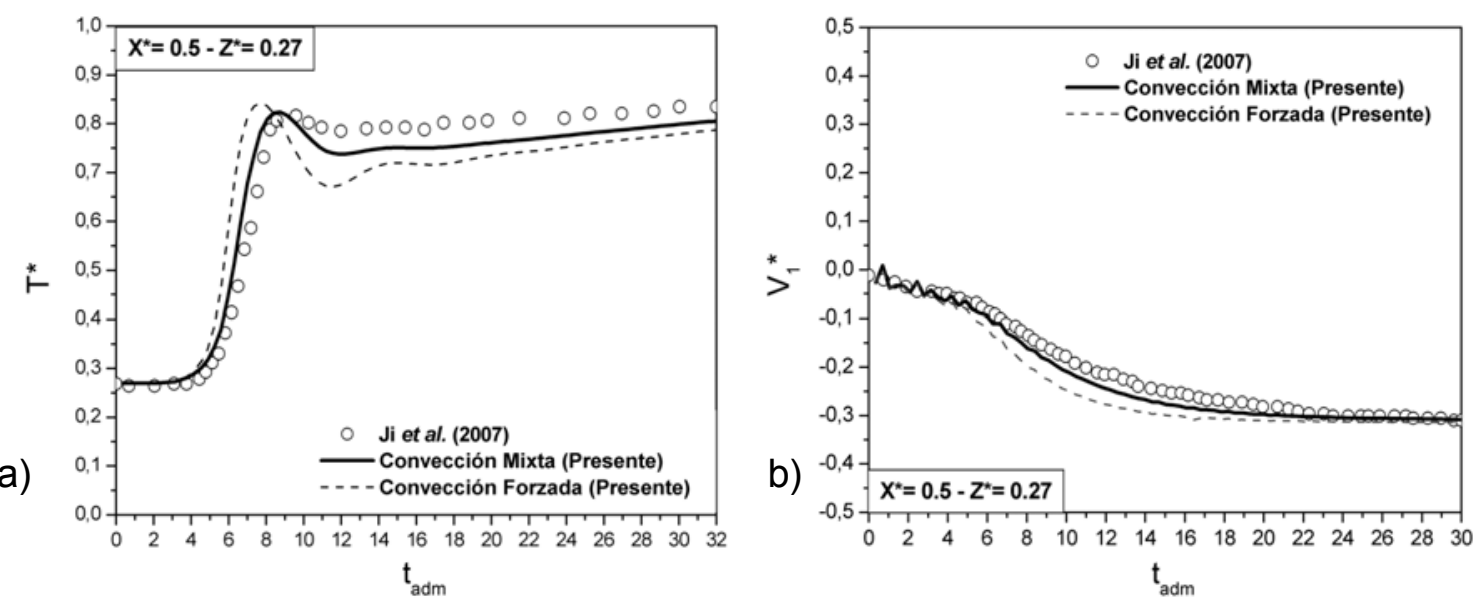

Fig. 7: Evolución temporal en $X=0,5$ y $Z=0,27-a)$ Temperatura - b) Velocidad en $X$ (adimensionales)
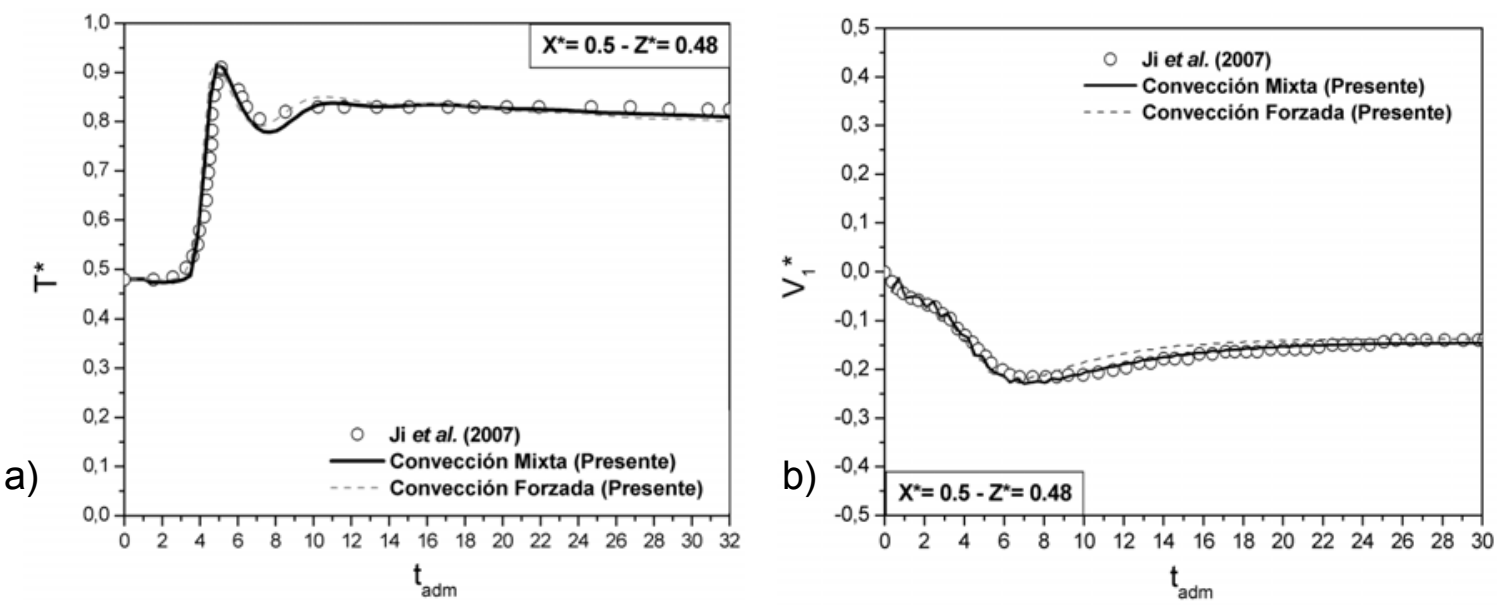

Fig. 8: Evolución temporal en $X=0,5$ y $Z=0,48-a)$ Temperatura - b) Velocidad en $X$ (adimensionales)
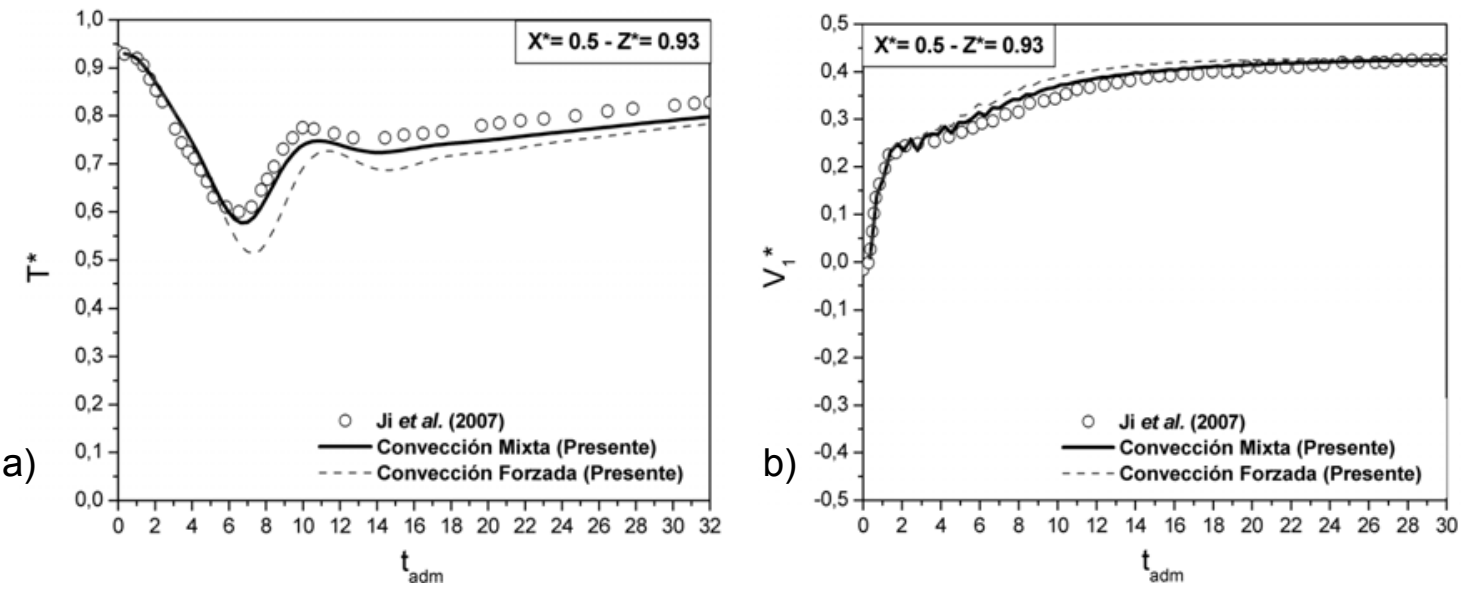

Fig. 9: Evolución temporal en $X=0,5$ y $Z=0,93-a)$ Temperatura - b) Velocidad en $X$ (adimensionales) 


\section{CONCLUSIONES}

En este trabajo fue presentado un estudio numérico sobre flujos turbulentos, estacionarios, en canales tridimensionales con $\operatorname{Re}=3300$ y flujos transitorios bidimensionales en cavidades con transferencia de calor por convección mixta $(\operatorname{Re}=400, \operatorname{Pr}=6$ y $\mathrm{Gr}=16000)$. La solución numérica fue basada en un código desarrollado en FEM, utilizando esquema temporal explicito de TaylorGalerkin. La turbulencia fue abordada a través de LES.

En la simulación de los flujos turbulentos en canales fueron comparados los dos modelos de submalla empleados. Se observó que los parámetros medios, así como las estadísticas de la turbulencia fueron mejor predichas en las simulaciones hechas con el modelo dinámico. El coeficiente de fricción predicho con los modelos dinámico y de Smagorisnky fueran 4,5\% y 16\%, respectivamente, más bajos que el predicho a través de DNS por (Kim et al., 1987). Con referencia a las estadísticas de la turbulencia, las tensiones no se previeron de manera satisfactoria con el modelo de Smagorinsky, lo que sugiere que la constante de submalla debería ser adaptable a lo largo del dominio. Esto tornase evidente cuando las tensiones son obtenidas a través del modelo dinámico con metodología del segundo filtrado propuesta por (Petry, 2002), visto que este modelo ha conducido a resultados más satisfactorios, reduciendo los problemas relacionados a selección del parámetro submalla.

Para abordar problemas con transferencia de calor fue inserida la ecuación de la energía y de los términos de flotabilidad en el código pre-existente en FORTRAN, que simulaba flujos isotérmicos, tridimensionales, incompresibles, en régimen laminar y turbulento. Para evaluación del código, fueron simulados flujos transitorios con transferencia de calor en una cavidad con estratificación estable. El término de flotabilidade fue evaluado mediante dos simulaciones, una con los términos desactivados (convección forzada) y otra con los términos activados (convección mixta). Las topologías del campo de temperaturas indican que el comportamiento fluido dinámico y térmico fue bastante coherente con el presentado en la literatura (Ji et al., 2007). Además, los campos transitorios de velocidad y temperatura en función del tiempo se compararon con los resultados de (Ji et al., 2007), obteniéndose un desvío inferior a 6\%. El próximo paso es realizar la validación del código desarrollado para flujos turbulentos y no isotérmicos utilizando LES.

\section{NOMENCLATURA}

C velocidad de propagación del sonido del medio $(\mathrm{m} / \mathrm{s})$

Cs constante de Smagorinsky

$\mathrm{C}(\mathrm{x}, \mathrm{t})$ coeficiente dinámico

$\mathrm{C}_{\mathrm{P}} \quad$ calor específico a presión constante $(\mathrm{J} / \mathrm{kg} \mathrm{K})$

$g_{i} \quad$ aceleración de la gravedad en la dirección i $(1,2 \circ 3)\left(\mathrm{m} / \mathrm{s}^{2}\right)$

$\mathrm{H} \quad$ altura de la cavidad $(\mathrm{m})$

k conductividad térmica del fluido $(\mathrm{W} / \mathrm{m} \mathrm{K})$

L longitud de la cavidad (m)

$\mathrm{Nu}$ número de Nusselt

$P$ presión $\left(\mathrm{N} / \mathrm{m}^{2}\right)$

$\operatorname{Pr} \quad$ número de Prandtl $(v / \alpha)$

$\mathrm{Pr}_{\mathrm{t}} \quad$ número de Prandtl turbulento

$\mathrm{q}_{\mathrm{cr}} \quad$ flujo de calor por convección y radiación $\left(\mathrm{W} / \mathrm{m}^{2}\right)$

q"' razón de generación de energía $\left(\mathrm{W} / \mathrm{m}^{3}\right)$

$\hat{q} \quad$ flujo de calor preescrito $\left(\mathrm{W} / \mathrm{m}^{2}\right)$

$\mathrm{Re}_{\mathrm{H}} \quad$ número de Reynolds $\left(\mathrm{U}_{\mathrm{o}} \mathrm{H} / \mathrm{v}\right)$

$\mathrm{S}_{\mathrm{ij}} \quad$ taza de deformación del campo de velocidades $\left(\mathrm{s}^{-1}\right)$

$\mathrm{T}$ temperatura $(\mathrm{K})$

$\mathrm{T}^{*} \quad$ temperatura adimensional $\left(\mathrm{T}-\mathrm{T}_{\min }\right) /\left(\mathrm{T}_{\max }-\mathrm{T}_{\min }\right)$

$\hat{T} \quad$ temperatura preescrita en el contorno $(\mathrm{K})$

$\mathrm{T}_{0} \quad$ temperatura evaluada en el estado de referencia $(\mathrm{K})$

$\mathrm{t}$ tiempo (s)

$t_{a d m} \quad$ tiempo adimensional $(\mathrm{tUo} / \mathrm{H})$ 
$t_{i} \quad$ valores prescritos de las fuerzas de superficie en el contorno $\left(\mathrm{N} / \mathrm{m}^{2}\right)$

$v_{i} \quad$ velocidades en las direcciones $i(i=1,2$ o 3 )

$v_{i}{ }^{*} \quad$ velocidades adimensional en las direcciones $i(i=1,2$ o 3$)$

$\hat{v}_{i} \quad$ velocidad prescrita en la dirección $\mathrm{i}(\mathrm{i}=1,2$ ou 3$)$

$x_{i} \quad$ coordenada en la dirección $i(i=1(x), 2(y)$ y $3(z))$

a difusividad térmica $\left(\mathrm{m}^{2} / \mathrm{s}\right)$

$\alpha_{\mathrm{t}} \quad$ difusividad térmica turbulenta $\left(\mathrm{m}^{2} / \mathrm{s}\right)$

$\beta \quad$ coeficiente de expansión volumétrica del fluido $\left(\mathrm{K}^{-1}\right)$

$Z^{*} \quad$ coordenada adimensional $(\mathrm{Z} / \mathrm{H})$

$\Gamma \quad$ contorno del dominio o de un elemento del dominio

$\delta_{i j} \quad$ delta de Kronecker

$\xi_{i}, \eta_{i}, \zeta_{i}$ coordenada normalizada o computacional en el nodo $\mathrm{i}$

$\eta_{j} \quad$ coseno director del vector normal al contorno en la dirección j

$\lambda \quad$ viscosidad volumétrica del fluido $(\mathrm{kg} / \mathrm{m} . \mathrm{s})$

$\mu \quad$ viscosidad dinámica del fluido $(\mathrm{kg} / \mathrm{m} . \mathrm{s})$

u viscosidad cinemática del fluido $\left(\mathrm{m}^{2} / \mathrm{s}\right)$

$v_{t} \quad$ viscosidad turbulenta $\left(\mathrm{m}^{2} / \mathrm{s}\right)$

$\rho \quad$ masa específica del fluido $\left(\mathrm{kg} / \mathrm{m}^{3}\right)$

$\rho_{0} \quad$ masa específica en el estado de referencia $\left(\mathrm{kg} / \mathrm{m}^{3}\right)$

$\sigma_{i j} \quad$ Fuerzas de superficies en el contorno $\left(\mathrm{N} / \mathrm{m}^{2}\right)$

\section{REFERENCIAS}

Ampofo, F., Karayiannis, T.G., Experimental benchmark data for turbulent natural convection in an air filled square cavity, Int. J. Heat Mass Transf.: 46, 3551 - 3572, 2003.

Abrunhosa, J.D.M., Simulação de Escoamentos Turbulentos Complexos com Modelagem Clássica e Simulação de Grandes Escalas, Tesis de Doctorado, Departamento de Ingeniería Mecánica, Pontificia Universidade Católica do Rio de Janeiro, Rio de Janeiro, Brasil (2003).

Azevedo, L. R., Analise de Problemas de Interação Fluido-Estrutura Usando o Método dos Elementos Finitos com um Acoplamento Monolítico, Tesis de Doctorado, Universidade Federal do Rio Grande do Sul, Porto Alegre, Brasil (1999).

Donea, J., A Taylor-Galerkin Method for Convective Transport Problems, Int. J. Numer. Methods Eng.: 20, 101-119 (1984).

Eiamsa-ard, S., Promvonge, P., Thermal Characteristics of Turbulent Rib-grooved Channel Flows, Int. Commun. Heat Mass Transf.: 36 (7), 705-711 (2009).

Germano, M., U. Piomelli, P. Moin y W.H. Cabot, A Dynamic Subgrid-Scale Eddy Viscosity Model, Phys. Fluids A: 3 (7), 1760-1765 (1991).

Gravilakis, S., Numerical Simulation of Low-Reynolds-Number Turbulent Flow Through a Straight Square Duch, J. Fluid Mech.: 244, 101-129 (1992).

Ji, T.H., S. Y. Kim, J. M. Hyun, Transient Mixed Convection in an Enclosure Driven by Sliding Lid, Heat and Mass Transf.: 43, 629-638 (2007).

Kawahara, M. y H. Hirano, A Finite Element Method for High Reynolds Number Viscous Fluid Flow Using Two Step Explicit Scheme, Int. J. Numer. Meth. Fluids: 3, 137-163 (1983).

Kim, J., P. Moin y R. Moser, Turbulence statistics in fully Developer channel flow at low Reynolds Number, J. Fluid Mech.: 177, 133-166 (1987).

Kobayashi, T., Large Eddy Simulation for Engineering Applications, Fluid Dyn. Res.: 38 (2-3), 84107 (2006) 
Lesieur, M., Métais, O., Comte, P., Large-Eddy Simulations of Turbulence, Cambridge, New York, USA (2005).

Lilly, D.K., A Proposed Modification of the Germano Subgrid-Scale Closure Method, Phys. Fluids A: 4 (3), 633-635 (1992).

Nishino, K., Kasagi, N., Turbulence Statistics Measurement in a Two-dimensional Channel Flow Using a Three-dimensional Particle Tracking Velocimetry, Proc. $7^{\text {th }}$ Symp. Turbulence Shear Flows, Stanford, 22.1.1-22.1.6, USA (1989).

Petry, A. P. y A. M. Awruch, Large Eddy Simulation of Three-Dimensional Turbulent Flows by the Finite Element Method, J. of the Braz. Soc. of Mech. Sci. \& Eng.: 28, 224-232 (2006).

Petry, A. P., Análise Numérica de Escoamentos Turbulentos Tridimensionais Empregando o Método de Elementos Finitos e Simulação de Grandes Escalas, Tesis de Doctorado, Universidade Federal do Rio Grande do Sul, Porto Alegre, Brasil (2002).

Reddy, J.N. y D.K. Gartling, The Finite Element Method in Heat Transfer and Fluid Dynamics, 1251, CRC, Boca Raton, USA (1994).

Saugat, P., Large Eddy Simulation for Incompressible Flows, An Introduction, Springer Berlin Heidelberg, Berlin, Germany (2006).

Silveira Neto, A., D. Grand, O. Métais, M. Lesieur, A Numerical Investigation of the Coherent Vortices in Turbulence Behind a Backward-Facing Step, J. Fluid Mech.: 256, 1-25 (1993).

Smagorinsky, J., General Circulation Experiment with Primitive Equations, I. The Basic Experiment, Mon. Weath.: 91, 99-164 (1963).

Wang, L., Y. Dong y X. Lou, An investigation of turbulent open channel flow with heat transfer by Large Eddy Simulation, Computers \& Fluids: 34, 23-47 (2005).

Yoon, K. T., S.Y. Moon, S.A. Garcia, G.W. Heard y T.J.Chung, Flowfield-dependent mixed explicitimplicit (FDMEI) methods for high and low speed and compressible and incompressible flows, Comput. Methods Appl. Mech. Engrg.: 151, 75-104 (1998).

Zienkiewicz, O.C. y R.L. Taylor, The Finite Element Method, 5 th Edition, Butterworth-Heinemann, Woburn, Massachussets, USA (2000). 